\title{
Influence of Doping on the Crystal Potential of Silicon investigated by the Convergent Beam Electron Diffraction Technique
}

\author{
R. Voss, G. Lehmpfuhl, and P. J. Smith * \\ Fritz-Haber-Institut der Max-Planck-Gesellschaft, Berlin \\ Z. Naturforsch. 35a, 973-984 (1980); received July 12, 1980
}

Low index structure potentials of silicon were determined by convergent beam electron diffraction (Kossel-Möllenstedt technique) from very small crystal areas of about $100 \AA$ in diameter. The values of $111,222,220,113$ and 004 , determined to an accuracy of \pm 0.03 volts, are in excellent agreement with the accurate X-ray results of Aldred and Hart (see [6], p. 239). Heavy arsenic or phosphorous doping was found to cause a shift of 0.15 volts in the 111 structure potential. Absorption potentials were also determined and found to be $1 / 3$ of the theoretical values published by Radi [20].

\section{Introduction}

Convergent beam electron diffraction technique developed by Kossel and Möllenstedt allows one to obtain information over an area of reciprocal space. This technique has recently been found to be a powerful tool in structure analysis work (see. e.g. $[1-3])$. The analysis of the diffraction pattern is done by means of the dynamical theory (following Bethe [4] or Cowley and Moodie [5]) using computer methods. In addition to information on crystal symmetry, low index structure amplitudes can be determined with an accuracy of better than $1 \%$. The low index structure amplitudes contain information on the interatomic electron density distribution, i. e. the distribution of the bonding electrons.

The reliability of the convergent beam technique can be shown by comparison of the structure amplitudes, determined by electron diffraction, with the very accurate $\mathrm{X}$-ray data obtained by Aldred and Hart [6]. In their investigation dynamical effects were used to determine structure amplitudes. The high accuracy they achieved was only possible because of the fact that silicon is available as large dislocation-free single crystals of several millimeter length.

In the present work, the structure amplitudes were determined from measurements on very small areas, $\sim 100 \AA$ in diameter. This determination from small areas has several advantages. For smaller

* Permanent adress: IBM, East Fishkill, New York, USA.

Reprint requests to Dr. G. Lehmpfuhl, Fritz-Haber-Institut der Max-Planck-Gesellschaft, Faradayweg 4-6, D-1000 Berlin 33 (Dahlem). areas there is greater likelihood of finding the required perfect single crystal areas, so that measurements can be made from materials which are not available as large single crystals. Also there is now a possibility for detecting variations in crystal potential on a micro-scale, as, for example, due to impurity variations in the neighborhood of dislocations or grain boundaries. An accurate determination of these structure amplitudes can become of great interest in the case of semiconductors such as silicon, as there arises the question of whether the influence of doping can be detected in electron diffraction intensity distributions. Results of such an investigation may provide a basis for an electron microscopic dark field technique to detect doping inhomogeneities near dislocations from such areas which are not yet disturbed by the strain field.

The purpose of our work was therefore twofold. Low index structure potentials were determined from lightly doped silicon and compared with previousX-ray measurements. Measurements were then made on heavily doped silicon to determine whether the technique was sensitive to variations in dopant concentration. In the present work we consider both the effect of non-systematic interactions and absorption, which were not included in a prior investigation [7].

\section{Theory}

For the analysis of the diffraction pattern densitometer records of the intensity distribution were compared with calculated intensities. An $n$-beam approximation of the dynamical theory of electron diffraction due to Bethe [4] was used to calculate

0340-4811 / $80 / 0900-0973 \$ 01.00 / 0$. - Please order a reprint rather than making your own copy. 
the intensities. The partial-wave amplitudes of the Bloch waves $(i)$ and their eigenvalues $\tau_{i}$ ("Anpassung") were calculated by solving an eigenvalue problem as described earlier [8] including the absorption by an imaginary part of the potential [9].

The intensity of a diffracted beam from a parallelsided crystal is given by the superposition of all partial waves $\psi_{g}{ }^{(i)}$ belonging to the index $g$ and different Bloch waves $(i)$ :

$$
I_{g}=\Psi_{g} \Psi_{g}^{*}
$$

where

$$
\begin{aligned}
& \Psi_{g}=\exp (2 \pi i \boldsymbol{g} \boldsymbol{r}) \underset{i}{\sum} \psi_{g}{ }^{(i)} \\
& \cdot \exp \left(-\frac{\mu_{i}}{2} t\right) \exp \left(\frac{2 \pi i}{\lambda} \tau_{i} t\right) .
\end{aligned}
$$

$t$ is the crystal thickness, $\boldsymbol{g}$ the reciprocal lattice vector and $\mathbf{r}$ a vector in the real space. The partial waves $\psi_{g}{ }^{(i)}$, the eigenvalues $\tau_{i}$ and the absorption coefficients $\mu_{i}$ of Bloch wave (i) depend on the crystal potential $V(r)=V_{\text {real }}+i V_{\text {im }}$ and on the wave vector $\boldsymbol{K}_{0}$ (direction and wave length $\lambda$ ) of the incident electron wave.

The real part of the silicon crystal potential was calculated from atomic scattering amplitudes tabulated by Doyle and Turner [10]. The influence of bonding is only found in the low index Fouriercoefficients of the crystal potential. So the coefficients as $111,222,220,311$ and 400 were used as adjustable parameters, starting with the DoyleTurner values.

The imaginary part, by which the absorption was considered, is related to the real part due to Humphreys and Hirsch [11] by the simple relation

$$
V_{g}{ }^{\text {im }}=V_{g}^{\text {real }}\left(B|\boldsymbol{g}|-C \boldsymbol{g}^{2}\right)
$$

with constants $B$ and $\mathrm{C}$ determined experimentally by Ichimiya and one of us [12] and refined during this analysis.

The direction of the incident electron beam was defined by the intersection of the Ewald sphere with the coordinate axes in the reciprocal lattice diffracting plane, i.e. points $\mathrm{G}$ and $\mathrm{H}$ in Figure 1 . They define the direction of the incident beam. In the calculation the influence of both systematic and accidential interactions of higher order Laue-Zones was included, i.e. for reflections from reciprocal planes parallel to the one shown in Figure 1. Contributions from both negative and positive Lauezones were included.

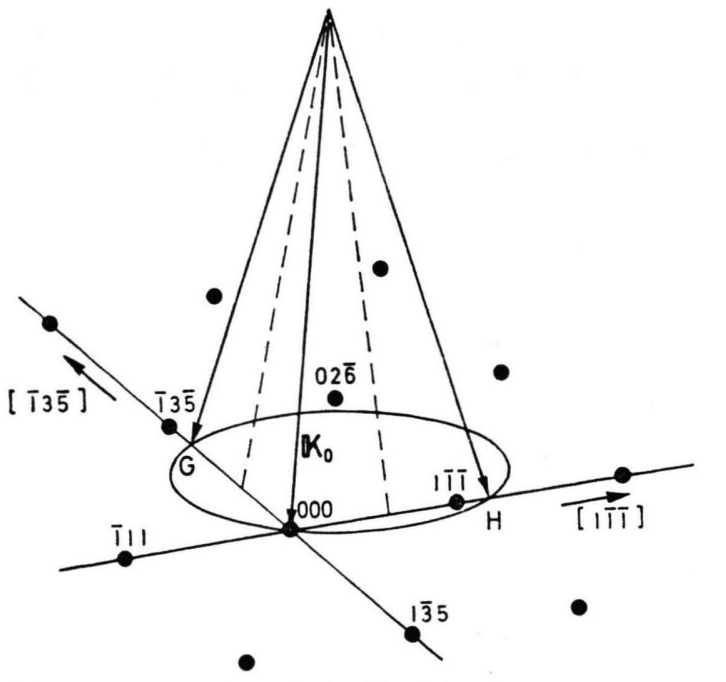

Fig. 1. Intersection of the Ewald sphere with a plane of the reciprocal lattice. The direction of the incident electron beam, characterized by the vacuum wave vector $\boldsymbol{K}_{0}$, is defined by the intersection points of the Ewald sphere with the two indicated axes. These points are $G$ and $H$.

\section{Experiment}

\section{Kossel-Möllenstedt technique}

In the convergent beam electron diffraction experiment (see e.g. [13]) the specimen is placed on a goniometer in the back focal plane of an objective lens. An aperture in front of the objective determines the cone angle of the electron beam which falls onto the specimen. The size of the aperture has to be chosen in such a way that the diffracted beams do not overlap. Such a diffraction pattern is called Kossel-Möllenstedt pattern.

The diameter of the electron beam on the specimen is of the order of $100 \AA$ using a strong objective lens and a small aperture limiting the cone angle to $10^{-2}$ rad. By defocusing the objective lens one obtains a shadow image of the specimen which allows the identification of the part from which the diffraction pattern comes. If the aperture in front of the objective is removed, electrons within a widely opened cone fall onto the specimen. The resulting diffraction pattern is called a Kossel pattern for electrons. Due to the spherical aberration of the objective lens the specimen area contributing to the diffraction pattern has then a diameter of $10-20 \mu$ depending on the convergent beam cone angle.

The experiments were done in a diffraction camera specially developed for application of the convergent beam technique. A detailed description 
will be given in a separate report. The camera was built up from commercial parts of the Siemens Elmiskop. The objective lens was used upside down. The specimen was inserted in the double tilt stage which was mounted on a lift to bring it into the back focal plane. Between specimen and photographic plate there were no lenses which is important for a quantitative analysis of the diffraction pattern.

\section{Contamination}

The specimen contamination, which occurs when working with a small electron probe under normal high vacuum conditions, could be avoided by a combination of special treatments with a cooled anticontamination shield surrounding the specimen. The method for reducing the contamination was described earlier [14]. It was found that a preillumination of the specimen with the widely opened convergent electron beam was the most effective treatment. The widely opened convergent beam is obtained by pulling out the aperture in front of the objective lens. Under this condition a specimen area of $10-20 \mu$ diameter, depending on the spherical aberration of the lens, is irradiated by the electrons. After two minutes the aperture is inserted again to obtain the Kossel-Möllenstedt pattern, which now can be observed for $60-75$ sec. without any remarkable contamination. After this period the contamination starts immediately. However, if one repeats the pre-illumination just before the end of this period no contamination occurs and after 1 minute again a contamination-free period can be used. This "interval technique" can be continued as long as necessary to investigate a small specimen area of about $100 \AA$ in diameter under normal high vacuum conditions without any distortion by contamination.

Before starting the very first pre-illumination with electrons the specimen on the goniometer stage is heated up to $200^{\circ}-250{ }^{\circ} \mathrm{C}$ for 2 minutes by an infrared heating lamp and a quartz light pipe in order to desorb hydrocarbon molecules. The heating lamp is outside the vacuum.

\section{Intensity measurement}

The diffraction pattern was recorded photographically. As reported earlier, Ilford films N4E.50 were used. Their sensitivity to electrons was calibrated by measuring the electron current density with a Faraday cup. The sensitivity of the film can be expressed by the number of electrons which are necessary to produce an optical density of 1.0. For the Ilford film N4E.50 this is just 1 electron $/ \mathrm{um}^{2}$ for $100 \mathrm{keV}$ electrons. The exposure time was controlled by a calibrated shutter. The intensity distribution in the diffraction pattern could then be determined by microdensitometer analysis of the photographic film.

\section{Sample preparation}

To determine the (111) structure potential, $V_{111}$, the crystal plate should have an orientation close to (110) so that the (111) reflection can be excited with minimal tilting. The crystal plates were cut from single crystal rods (Wacker Chemie) parallel to the (110) plane. They were etched and polished prior to thinning for examination in the convergent beam camera. Crystals with the following doping were examined:

(1) pure $\mathrm{Si}\left(10^{13} / \mathrm{cm}^{3}\right.$ of $\mathrm{B}$, float zone),

(2) Si with $0.012 \% \mathrm{P}\left(6 \times 10^{18} / \mathrm{cm}^{3}\right.$, Czochralski $)$,

(3) Si with $0.1 \%$ As $\left(5 \times 10^{19} / \mathrm{cm}^{3}\right.$, Czochralski).

Attempts to investigate $\mathrm{Si}$ with high boron doping $\left(1 \times 10^{19} / \mathrm{cm}^{3}\right)$ were unsuccessful due to the uneven etching during sample preparation. Some (100) and (111) plates were also prepared and examined.

\section{Experimental procedure}

Kossel-Möllenstedt diffraction patterns from (111) lattice planes were taken far away from the [110] zone axis to keep the influence of nonsystematic interactions as small as possible. In a first approximation the diffraction pattern was considered to show only systematic interactions. The accidental interactions could be recognized as dark or bright lines crossing the convergent beam diffraction disks. In order to find a proper orientation, a Kossel panorama was recorded by a sequence of Kossel patterns. Starting with a Kossel pattern around the [110] zone axis the following Kossel patterns were taken each after rotating the crystal about the [T11] axis in steps of $8^{\circ}$. Such a Kossel panorama, which is similar to the Kikuchi panorama, is shown in Figure $2 \mathrm{~b}$. From this panorama it is easy to find an orientation of the crystal where the influence of accidental interactions is small. Together 
with the Kossel panorama two Kossel-Möllenstedt patterns are shown: the one with the [110] zone axis in the center of the zero beam and the other with the systematic (T11) excitation near the [431] zone axis.

The orientation of the crystal can be determined from a Kossel pattern by comparing the Kossel panorama in Fig. $2 \mathrm{~b}$ with a calculated system of Kikuchi lines shown in Figure $2 \mathrm{a}$. By this comparison wave length and instrument camera length could be determined independently within an accuracy of $1 \%$.

Kossel-Möllenstedt patterns with strongly excited (III) and $(2 \overline{2} \overline{2})$ reflections were taken near the [431] zone axis where the influence of accidental interaction is small. Exposures with several different exposure times were necessary due to the large intensity difference in the different reflections. Kossel-Möllenstedt patterns with (1TI) excitation are

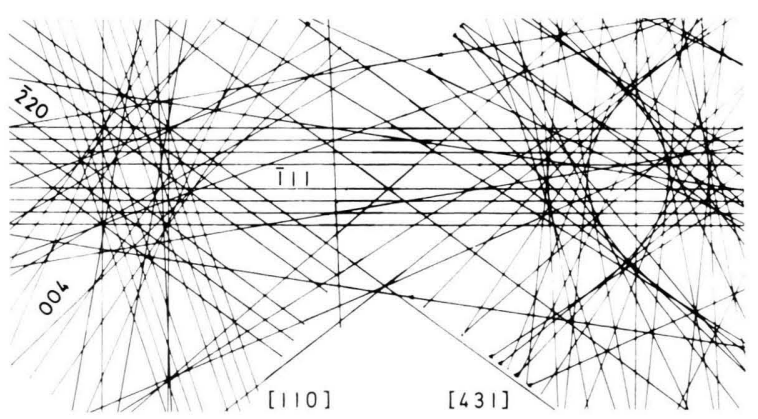

a)
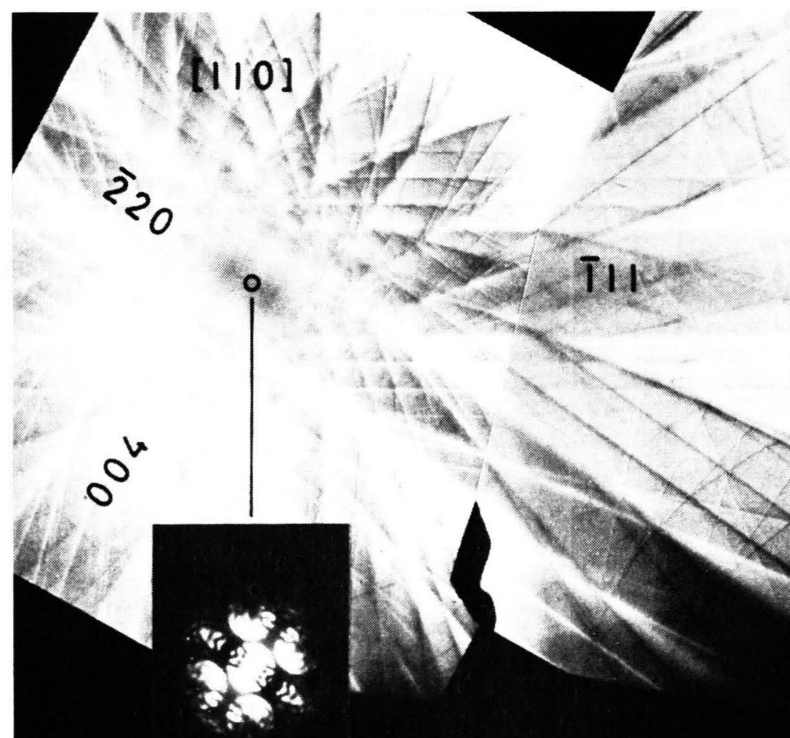

) shown in Fig. 3 for three different exposure times. The long exposure was necessary to determine the inelastic background and to see the weak systematic reflections. The intensity distribution in the diffraction pattern of Fig. 3 along the indicated straight trace line in the top frame was obtained photometrically using the Joyce Loebel microdensitometer MK 111 and is shown in Fig. 4 a. The intensity distribution shows a background.

\section{Determination of the Inelastic Background}

The intensity distribution in Fig. 4 a consists of the intensity due to both elastic and inelastic scattering. The inelastic background has to be subtracted from the densitometer record to obtain the elastic diffraction intensities which can be compared with the calculations. To determine the background densitometer traces were taken along a maximum

Fig. 2. Kossel pattern of silicon around [110] and [431] zone axes. a) Calculation, b) Experiment. In b) Kossel-Möllenstedt patterns are inserted for the two zone axes.

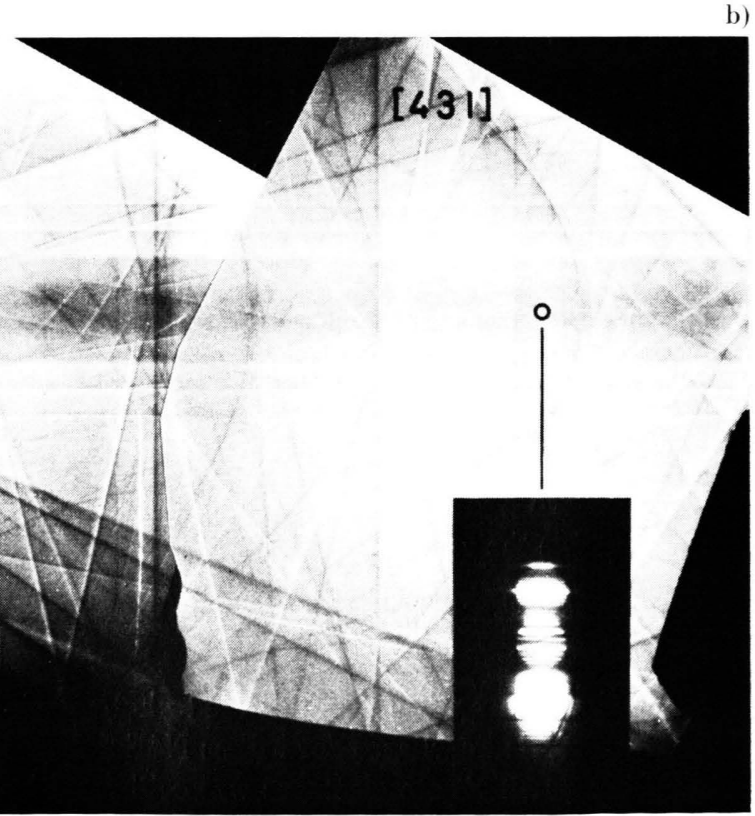



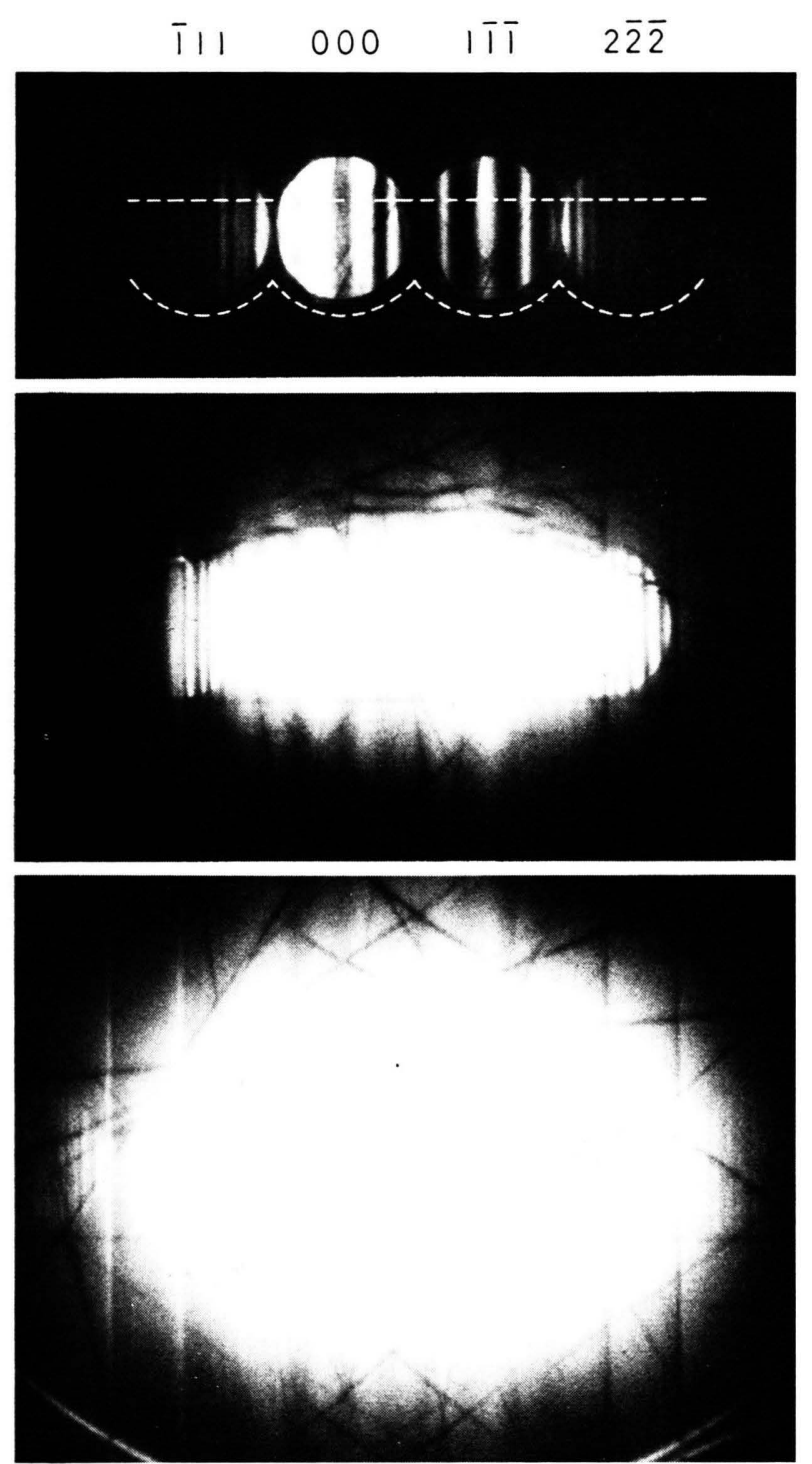

Fig. 3. Kossel-Möllenstedt patterns of $1 \overline{1} \overline{1}$-systematic interaction near the [431] zone axis taken with different exposure times (from top: $1 \mathrm{sec} ; 3 \mathrm{sec} ; 15 \mathrm{sec}$ resp.).

or a minimum in the diffraction disk perpendicular to the straight line indicated in the top frame of Figure 3. Figure $5 \mathrm{~b}$ shows a typical densitometer trace along a maximum as indicated in Fig. 5 a by the dashed line. The intensity distribution consists of the intensity of the elastically scattered electrons which is assumed to be constant in a systematic case, and the variable inelastic background. This is shown schematically in Figure $5 \mathrm{c}$. The right part of the densitometer curve in Fig. $5 \mathrm{~b}$ is disturbed by four Kikuchi lines while the left part is nearly undisturb-

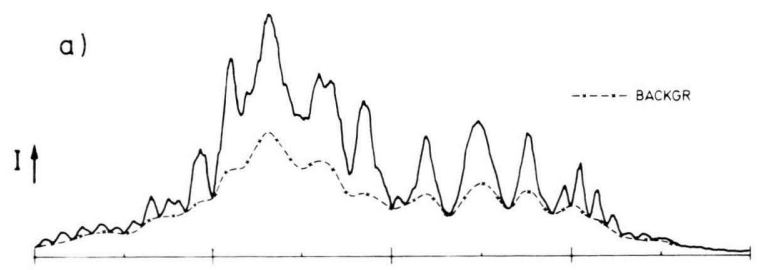

b)
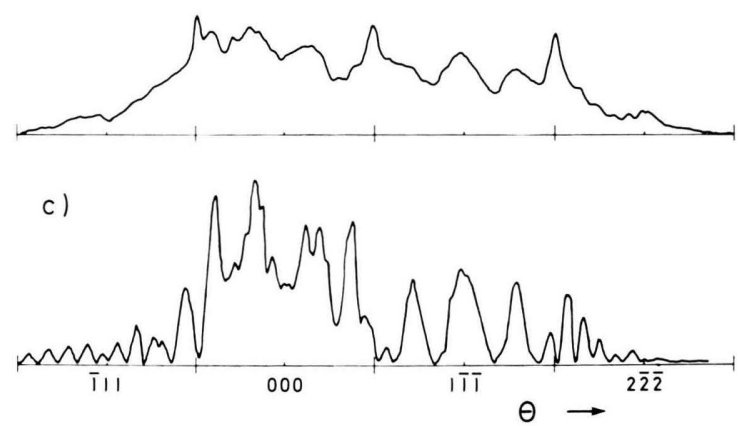

Fig. 4. a) Densitometer record of the intensity distribution in the Kossel-Möllenstedt pattern of Fig. 3 along the straight indicated dashed line with background. The crosses are determined from measurements explained by Figure 5. b) Background distribution along the dashed curved line outside the diffraction disks in Figure 3. c) Elastic intensity distribution from (a) after substracting the background (b).

ed, except by a line at the edge. The Kikuchi lines can be seen in the enlarged Kossel-Möllenstedt diffraction disk in Figure 5 a. The area from which the densitometer trace was obtained was always checked by such enlargements to see the influence of accidental interactions, as indicated by the presence of Kikuchi lines.

In this way the background was determined for each maximum and minimum. The values were transferred into the densitometer records as shown in Fig. 4 a by the crosses. Especially in the minima the background was measured very carefully to see if the elastic intensity was really zero or if there was still a remaining contribution; this was very important for the curve fitting procedure during the analysis.

The background between the measured points was determined by a densitometer record outside the diffraction disks along a trace indicated in Fig. 3 and is shown in Figure $4 \mathrm{~b}$. This curve was obtained from the diffraction pattern with the longest exposure time in Figure 3. The resulting background is shown in Figure $4 \mathrm{a}$. The determination of the background could be simplified by using a rectangu- 
a)

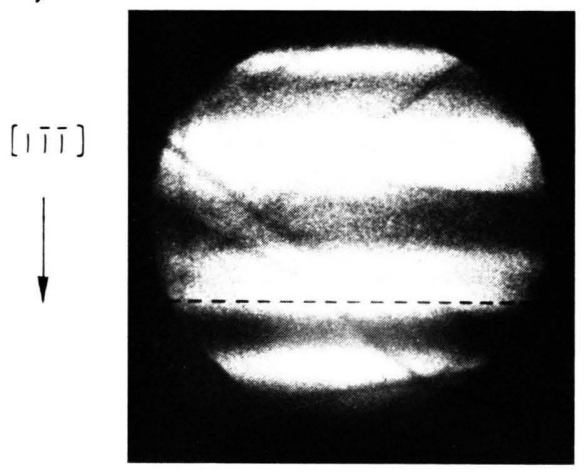

b)

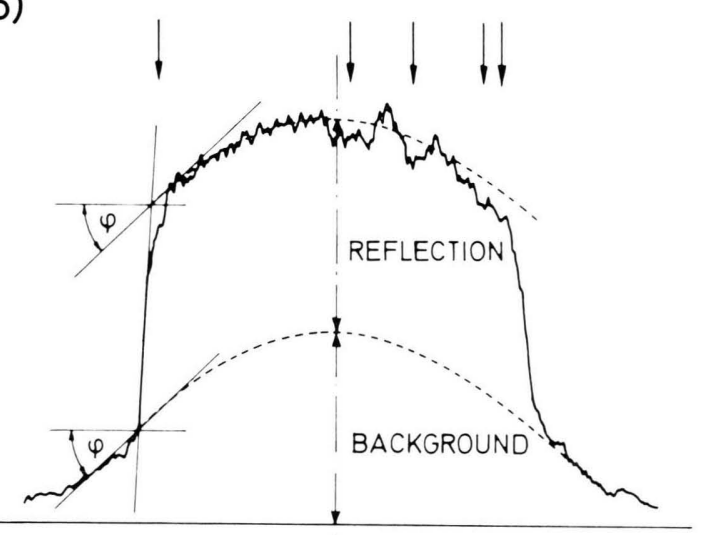

c)

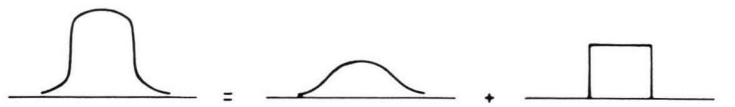

MEASURED

INELASTICAL

INTENSITY
BACKGROUND
ELASTICAL

REFLECTION

Fig. 5. Determination of the inelastic background. a) Kossel-Möllenstedt diffraction disk 000 . b) Densitometer trace along an intensity maximum indicated by the dashed line in (a). c) Schematic separation of the intensity into elastic and inelastic contributions.

lar aperture with one side parallel to the diffraction direction, as was done by M. Tanaka (private communication).

The elastic intensity distribution was obtained after digitizing the densitometer record of the diffraction pattern and subtraction of the digitized background. The resulting intensity distributions as shown e. g. in Fig. 4 c were used for the analysis.

\section{Results}

The experimental intensity distributions were compared with calculated intensities. Values for the thickness, orientation, wave length, camera length, low index structure potentials and absorption potential

were used as parameters in the calculation. The calculation was started with an estimated set of parameters using the two-beam approximation for thickness, a calculated Kikuchi pattern, the structure potentials due to Doyle and Turner [10] and absorption values published by Ichimiya and one of us [12]. These parameters were refined during the curve fitting process. Since the parameters are not independent of each other they had to be varied consistently.

The initial calculations included only systematic interactions [21]. In this one-dimensional calculation it is not necessary to know the exact orientation of the crystal. The intensity profiles in the different reflections depend in a characteristic way on structure potentials, absorption potential and crystal thickness. The profile in the center of the strong (III) beam depends mainly on the (111) structure potential, the asymmetry in the zero beam depends on the absorption potential and the profiles in the weak beams depend on thickness. Especially the distance between two maxima, the one very left and the other very right (e.g. in $\overline{1} 11$ and $2 \overline{2} \overline{2}$ of Fig. 4 a resp.), depends very sensitively on thickness. The high accuracy of the analysis is achieved by comparison of a continuous plot of the intensity distribution over all visible diffraction disks with calculated intensity distributions. Examples are given in Figs. 6, 7 and 8 for the influence of the structure potential, the absorption potential and the thickness, respectively, on the intensity profiles. The parameters are not independent of each other as mentioned above, so one observes dynamic effects in the strong reflections, which are very thicknesssensitive. Such dynamic effects can be seen in Fig. 8 for the relative height of the two maxima (1) and (2). The intensity profiles are calculated for different thicknesses in steps of 1 lattice constant. By the curve fitting procedure the crystal thickness could be determined within an accuracy of one lattice constant. This implies that the crystal thickness had to be constant over the illuminated area of $100 \AA$ in diameter. 


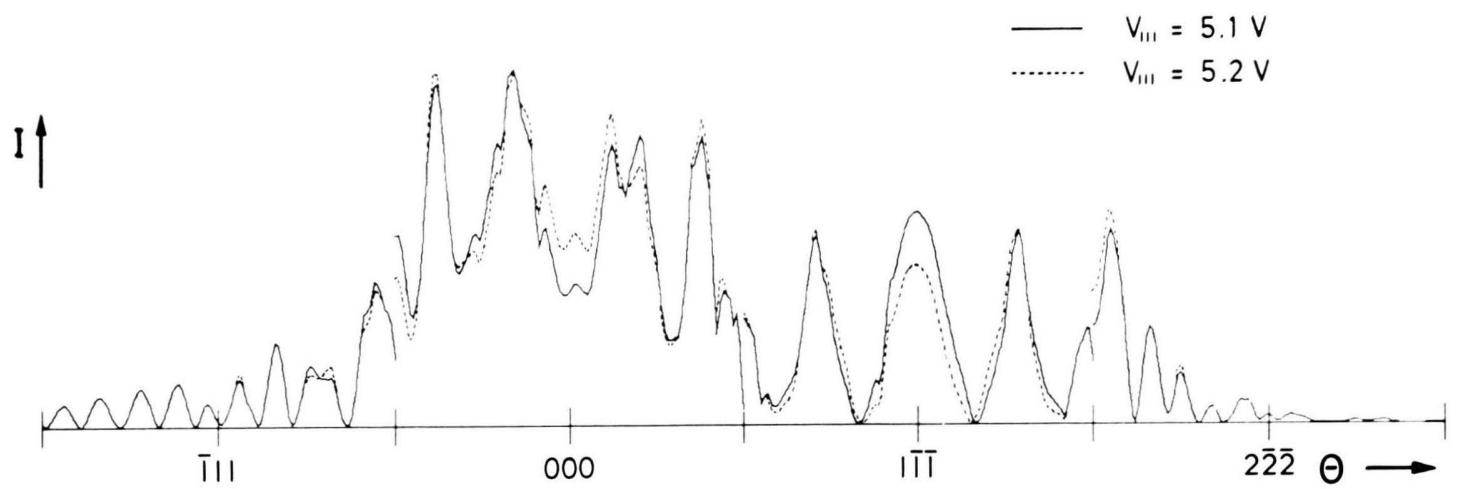

Fig. 6. Influence of the 111 structure potential on the calculated intensity distribution in the Kossel-Möllenstedt pattern for $1 \overline{1} \overline{1}$ excitation.

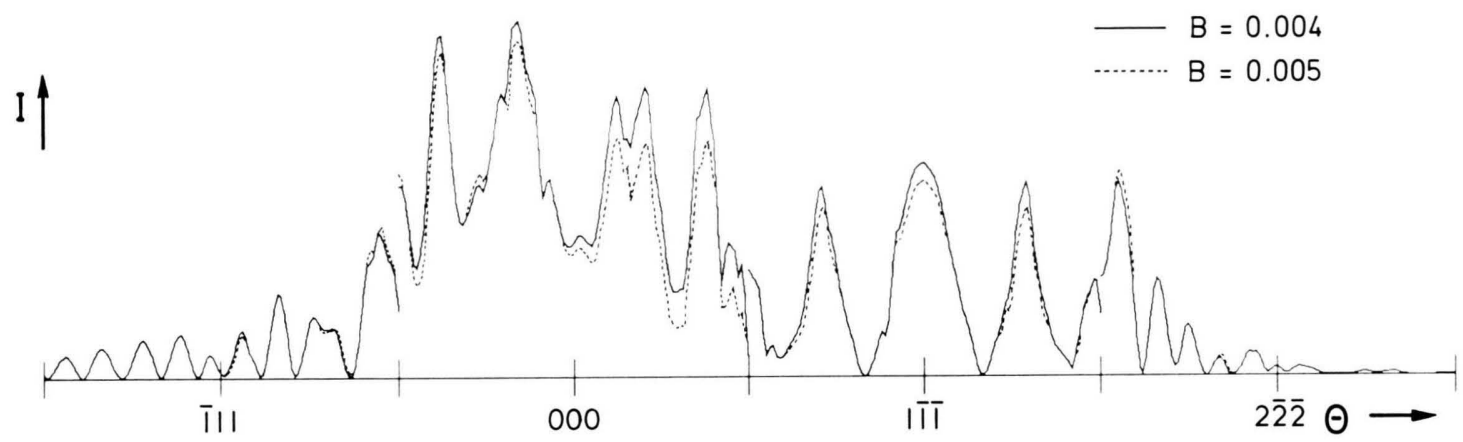

Fig. 7 Influence of the absorption potential - characterized by B - on the calculated intensity profile in the KosselMöllenstedt pattern of $1 \overline{1} \overline{1}$ excitation.

14

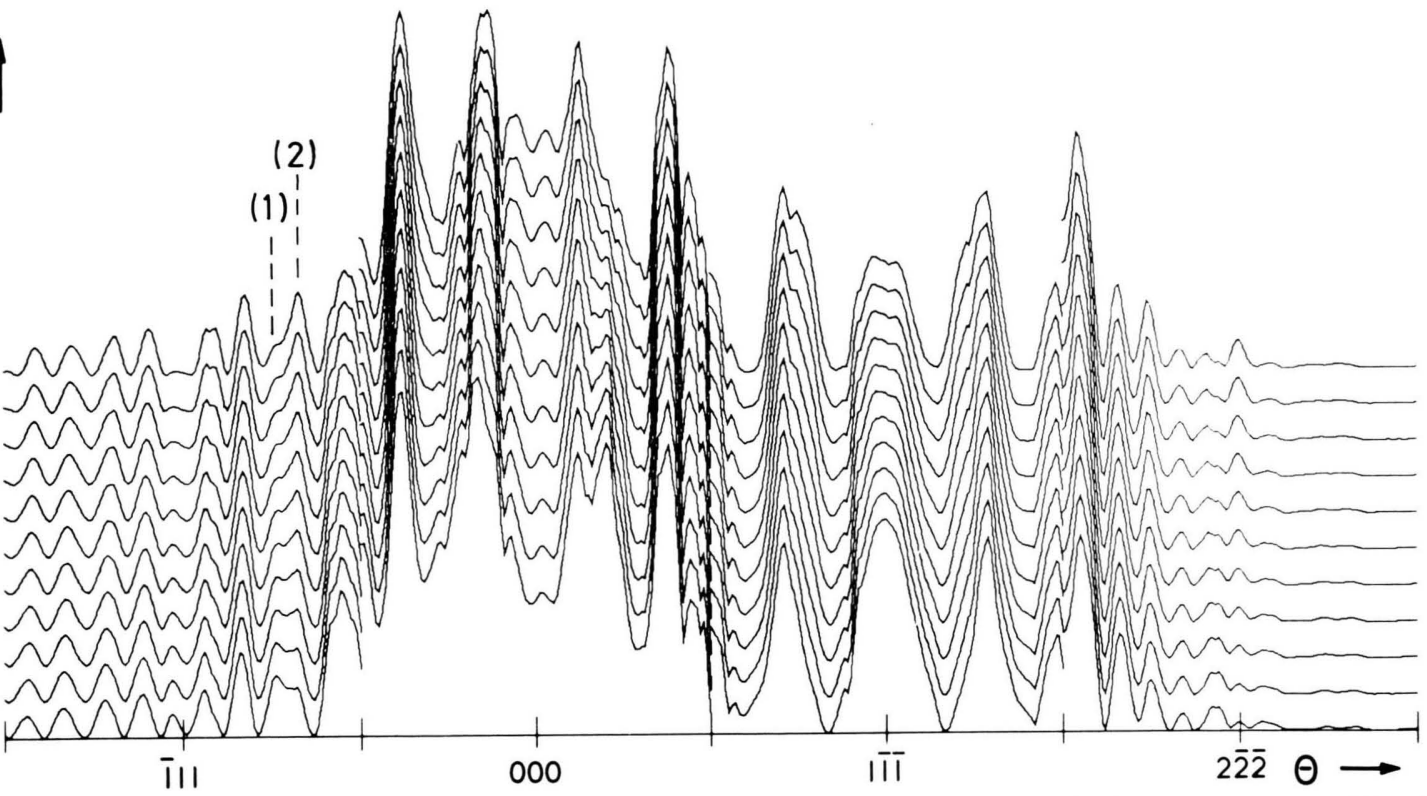

Fig. 8. Influence of the crystal thickness on the intensity distribution of the Kossel-Möllenstedt pattern. The thickness increases from the bottom to the top in steps of $5.43 \AA$, the lattice constant of silicon. (1) and (2) indicate two maxima showing very sensitive dynamic thickness effects. 
The wave length of the electrons was determined from the intersection of Kikuchi lines which belong to different zone axes. The area of a small triangle which is formed by three lines shown in Fig. 9 a by the arrow in the 000-diffraction disk is sensitive to the wave length. In a first approximation a geometric analysis can be done to determine the energy of the electrons with an accuracy of $1 \mathrm{keV}$. The intersecting Kikuchi lines, however, are not straight but are bent due to dynamic effects. To account for this effect full 2-dimensional dynamic many-beam calculations are required. Such 2-dimensional calculations are necessary for the determination of the exact crystal orientation. As was mentioned above, the crystal orientation is defined by the intersection points of the Ewald-sphere with the two vectors $\boldsymbol{G}$ and $\boldsymbol{H}$ shown in Figure 1. $\boldsymbol{G}$ and $\boldsymbol{H}$ define the plane of the reciprocal lattice containing all reflections of the zero order Laue-zone. For the analysis of the (1Ti) systematics the $[1 \overline{1} \overline{1}]$ direction was chosen as the vector $\boldsymbol{H}$. During the calculation $\boldsymbol{H}$ changed from 0.0 to 2.0 for the (1TI) excitation and from 1.0 to 3.0 for the $(2 \overline{2} \overline{2})$ excitation. The interference (1IT) is exactly excited at $\boldsymbol{H}=1.0,(2 \overline{2} \overline{2})$ is excited at $\boldsymbol{H}=2.0$ etc. For the [431] zone axis the reciprocal lattice vector $[\overline{1} 3 \overline{\overline{5}}]$ was chosen as $\boldsymbol{G}$. The accidental interaction in the experiment was then defined by the intersection point of the Ewaldsphere with this axis. For the 2-dimensional calculation a sequence of calculations was carried out, with $\boldsymbol{H}$ varying from 0.0 to 2.0 for different values of $\mathbf{G}$, in steps of $1 G=0.03$.

From such a set of calculations the intersecting Kikuchi lines in the reflection disks of a KosselMöllenstedt pattern were determined and compared with the experiment as shown in Figure 9. From this comparison the orientation could be determined within an accuracy of $4 G= \pm 0.03$ and the value for the accelerating voltage refined to $\pm 500 \mathrm{~V}$.

The influence of accidental interactions on the intensity profile for two different $\boldsymbol{G}$ values is shown in Figure 10. For the analysis a sequence of densitometer traces at different $\boldsymbol{G}$ values was compared with such calculations. These curves are compared with only systematic interactions. For the systematic calculation the interaction of 7 beams was taken into account.

One result of the curve fitting procedure is shown in Fig. 11 for 1T̄. By this example the degree of agreement between calculation and experiment is

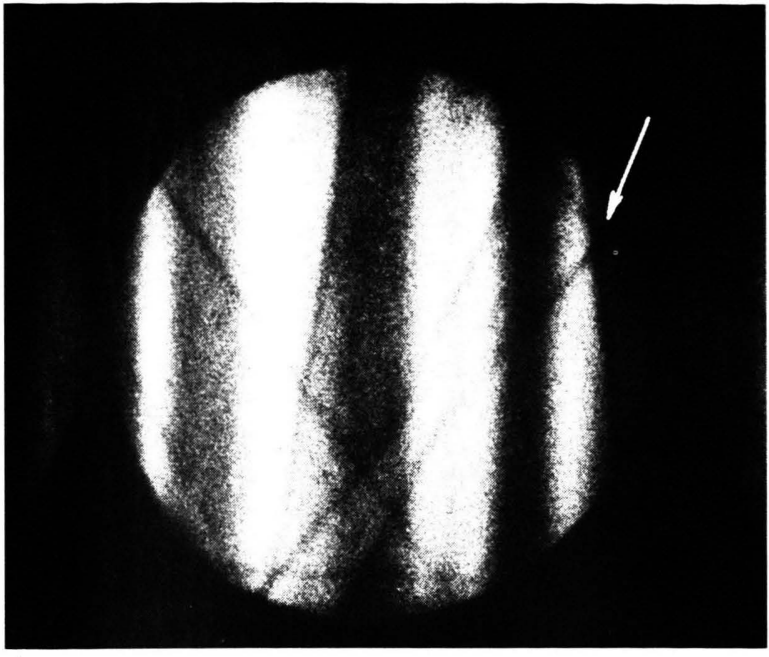

\section{a)}

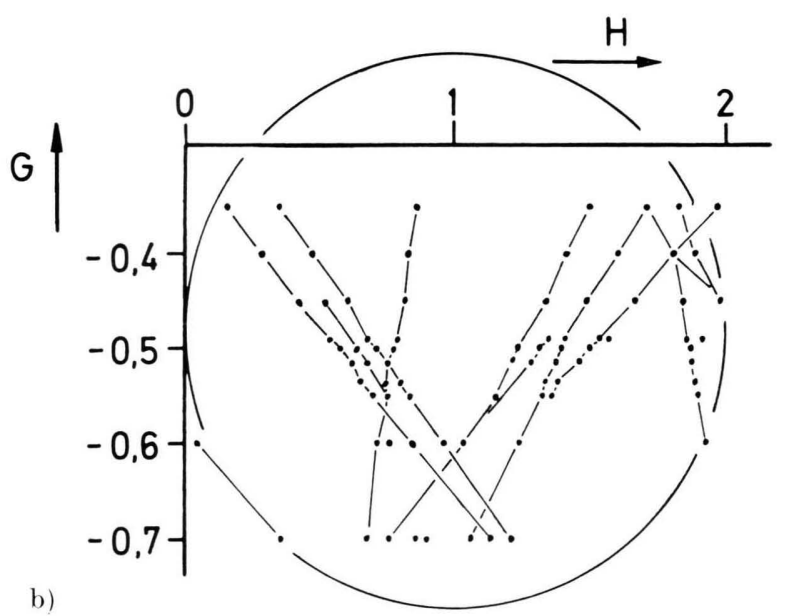

Fig. 9. Kikuchi lines in the Kossel-Möllenstedt 000-diffraction disk; a) experiment, b) from 40-beam calculations as shown in Fig. 10 at orientations indicated by $\mathrm{G}$ and $\mathrm{H}$.

shown. For the calculated intensity distribution accidental interactions from different Laue-zones were taken into account in a 40-beam calculation. For the intensity distribution in 220 and in 400 and also in 311 only systematic interactions were taken into account. That means that the corresponding structure potentials $V_{220}, V_{400}$ and $V_{311}$ were determined independently from each other and from the structure potentials $V_{111}$ and $V_{2222}$.

In Table 1 the experimentally determined structure potentials from pure silicon at room temperatur are shown together with the theoretical values due to Doyle and Turner [10], which are corrected for room temperature. In Table 2 the structure 

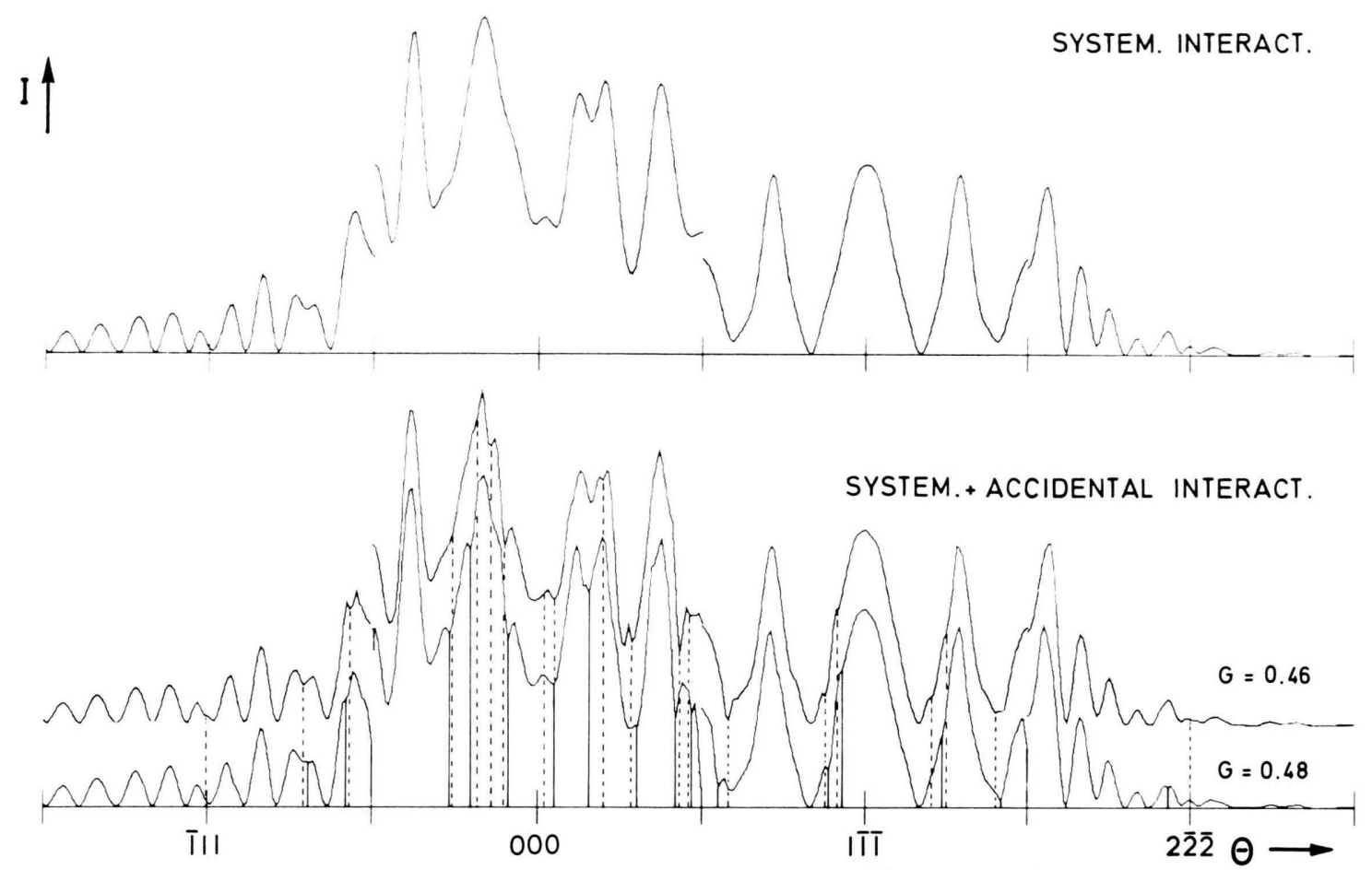

Fig. 10 Intensity distribution in the Kossel-Möllenstedt pattern with $1 \overline{1} \overline{1}$ excitation for two slightly different conditions of accidental interactions which are characterized by G. In the 40-beam calculation reflections from different Laue zones had been taken into account. Details of a fine-structure of the profile change with the direction of the incident beam. The influence of the accidental interactions can be seen by comparison with a calculation considering only systematic interaction of 7 beams.

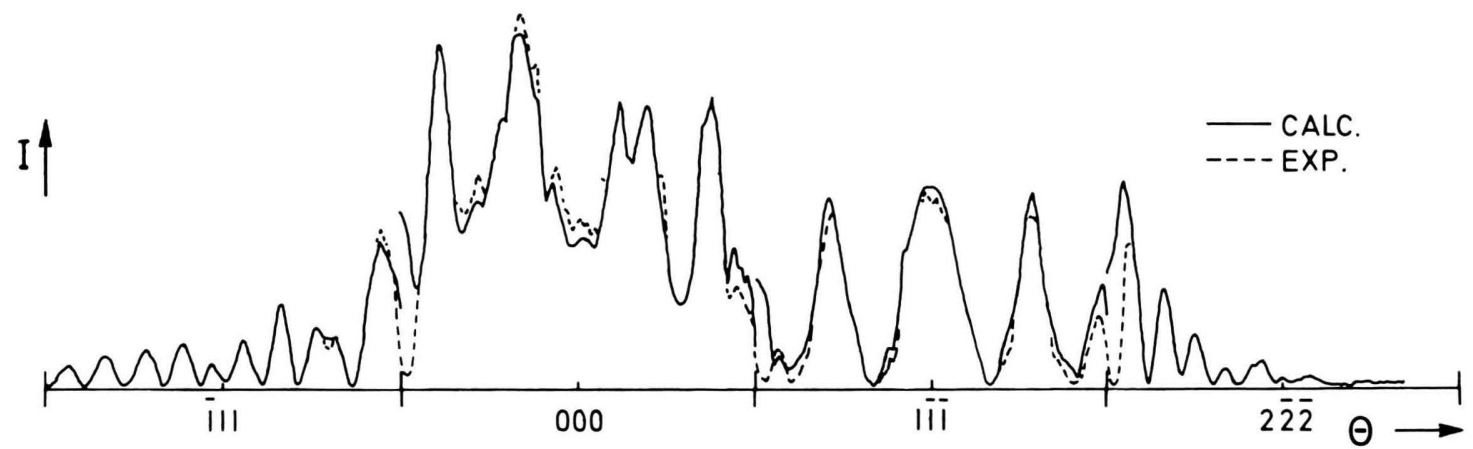

Fig. 11. Comparison of the experimental intensity distribution in the $1 \overline{1} \overline{1}$ Kossel-Möllenstedt diffraction patterns with a calculation.

potential $V_{111}$ for heavily doped silicon at room temperature is compared with the value for the pure specimen. As mentioned above it was not possible to investigate a heavily boron-doped silicon crystal, since the uneven etching made it impossible to find sufficiently large $(100 \AA)$ areas of uniform thickness.

The parameters of the absorption potential were determined consistently for all investigated speci- mens as

$$
B=0.004 \quad \text { and } \quad C=0.0003
$$

and a mean absorption potential of

$$
V_{000}^{\mathrm{im}}=0.61 \text { Volt. }
$$

The mean absorption potential was determined by measuring the intensity of the incident electron beam with a Faraday cup, the intensity distribution 
Table 1. Structure potentials of weakly doped silicon.

\begin{tabular}{lllr}
\hline$h k l$ & Experiment & $\begin{array}{l}\text { Calculation } \\
\text { (Doyle and } \\
\text { Turner 1968) } \\
\text { (Volt) }\end{array}$ & $\begin{array}{l}\text { Differ- } \\
\text { ence }\end{array}$ \\
\hline 111 & $-5.12 \pm 0.03$ & -5.43 & -0.31 \\
220 & $-4.40 \pm 0.03$ & -4.337 & 0.06 \\
311 & $-2.50 \pm 0.03$ & -2.435 & 0.07 \\
222 & $-0.15 \pm 0.03$ & 0 & -0.15 \\
400 & $-2.60 \pm 0.03$ & -2.566 & 0.03 \\
\hline
\end{tabular}

\begin{tabular}{ll}
\hline Doping & $\begin{array}{l}V_{111} \\
(\text { Volt })\end{array}$ \\
\hline $1 \cdot 10^{13}$ B-atoms $/ \mathrm{cm}^{3}$ & -5.12 \\
$6 \cdot 10^{18} \mathrm{P}$-atoms $/ \mathrm{cm}^{3}$ & -5.28 \\
$5 \cdot 10^{19} \mathrm{As}$-atoms $/ \mathrm{cm}^{3}$ & -5.25 \\
$1 \cdot 10^{19} \mathrm{~B}$-atoms $/ \mathrm{cm}^{3}$ & - \\
\hline
\end{tabular}

Table 2. Influence of doping on the 111 structure potential of silicon.

in the diffraction pattern from the calibrated density of the phhotographic film and a very accurate value for the crystal thickness.

\section{Discussion}

The accuracy of our structure potential measurements can be demonstrated by comparing our values with the very accurate $\mathrm{x}$-ray structure factors determined by Aldred and Hart [6]. For this comparison our structure potentials have to be converted to the $\mathrm{x}$-ray structure factors by the relation

$$
\begin{aligned}
& F_{h k l}^{\mathrm{x}}=Z 2 \cos \frac{\pi}{4}(h+l i+l) \\
& \text {. }\left[1+e^{2 . \pi i(h+h)}+e^{2 . x i(h+l)}+e^{-2 . i(l+h)}\right] \\
& -\frac{\pi a\left(h^{2}+k^{2}+l^{2}\right)}{300 e} V_{\text {hlil }}
\end{aligned}
$$

with $Z$ the atomic number, $a$ the lattice constant, $e$ the electron charge and $V_{h k l}$ the structure potential in volts. The zero point of the atomic lattice is chosen halfway between two closest silicon atoms. The sign of some structure potentials depends on the position of the zero point.

The 222 interference is forbidden due to the structure factor, but by "Umweganregung" this reflection can be excited, as is shown by a dynamical n-beam calculation. However, the intensity distribution in the Kossel-Möllenstedt pattern of the (111) systematic excitation can only be correctly re- produced by introducing a value for the 222 structure potential given in Table 1 . This value had to be always negative, independent of the position of the zero point of the atomic lattice, which means the sign of the other structure potentials. This leads to a positive x-ray structure factor $F_{222}^{\mathrm{x}}>0$ which is due to the bonding electrons.

In Table 3 our values are compared with the x-ray structure factors from Aldred and Hart [6], corrected for the temperature factor $\exp \{-B(\sin \Theta \mid$ i.) ${ }^{2}$ with $B=0.4613$ to obtain the stationary values. The agreement is much better than $1 \%$ except for the 222 value, which was measured directly only by electron diffraction while the $\mathrm{x}$-ray value was indirectly derived from the other structure factors using Dawson's method. The accuracy of the converted electron diffraction values is also better than $1 \%$. This shows the reliability of our structure potential measurements from very small crystal areas of the order of $100 \AA$ in diameter and the measured influence of doping on the 111-structure potential shown in Table 2. The deviation of the structure factors from the Doyle-Turner values is due to the bonding.

The high accuracy of structure factor determination by x-ray diffraction was only possible because of the availability of large perfect silicon single crystals. To measure the x-ray structure factors Aldred and Hart had to prepare separate crystals with different well defined surface orientations for each value $(h k l)$. Our neasurements were all taken from one parallel-sided crystal plate with (110) surfaces. Similar results were obtained from crystals with parallel (100) and (111) faces.

In Table 4 we compare our results with electron diffraction measurements of other authors. The agreement is reasonably good, supporting the reliability of the electron diffraction method. As

Table 3. Comparison of the present results with $\mathrm{x}$-ray struc-

\begin{tabular}{|c|c|c|c|}
\hline$h k l$ & present result & $\begin{array}{l}\text { x-ray mea- } \\
\text { surement } \\
\text { (Aldred and } \\
\text { Hart 1973) }\end{array}$ & $\begin{array}{l}\text { Differ- } \\
\text { ence }\end{array}$ \\
\hline 111 & $60.80 \div 0.11$ & 60.75 & $0.2 \%$ \\
\hline 220 & $69.00-0.28$ & 69.21 & $0.3^{\circ} \mathrm{o}$ \\
\hline 311 & $45.21 \pm 0.39$ & 45.39 & $0.4^{\circ}$ \\
\hline 222 & $2.23 \div 0.43$ & 1.42 & - \\
\hline 400 & $59.53 \pm 0.57$ & 59.5 .5 & $0.03 \%$ \\
\hline
\end{tabular}
ture factors due to Aldred and Hart [6] devided by the Debye-Waller factor with $B=0.4613$ at $293.2 \mathrm{~K}$. 
Table 4. Comparison of the present results with other electron diffraction measurements. The structure potentials are given for room temperature, except the values from Kreutle and Meyer-Ehmsen [16]. For the comparison only the absolute values of the structure potentials and structure factors of 111 and 220 were considered. However, the 222 structure potential values are compared with their sign. They are negative, independent of the direction of the vector 222 . Consequently the converted x-ray values $F_{222}^{\mathbf{x}}$ are positive. The values of Kreutle and Meyer-Ehmsen [16] and Ando et al. [17] seem to be absolute values. We may assume because of their accurate 111 structure potential that they didn't care at all about the sign of the structure potentials. The 222 structure potential of Shishido and Tanaka [18] was recalculated from their experimentally determined relation between $f_{111}$ and $f_{222}$ assuming $V_{111}=$ 5.12. The structure potentials of Terasaki et al. [15] were recalculated from their averaged $F^{\mathbf{x}}$-values. The $\mathrm{F}^{\mathbf{x}}$-values are stationary values (devided by the Debye Waller factor).

\begin{tabular}{|c|c|c|c|c|c|c|}
\hline & $V_{111}$ & $V_{222}$ & $V_{220}$ & $F_{111}^{\mathrm{x}}$ & $F_{\mathbf{2 2}}^{\mathbf{x}}$ & $F_{\mathbf{2 3 0}}^{\mathbf{x}}$ \\
\hline $\begin{array}{l}\text { Kreutle } \\
\text { et al. }(1969) \\
\left(500^{\circ} \mathrm{K}\right)\end{array}$ & 5.16 & 0.10 & - & & & \\
\hline $\begin{array}{l}\text { Ando et al. } \\
\text { (1974) }\end{array}$ & 5.13 & 0.12 & - & & & \\
\hline $\begin{array}{l}\text { Smith et al. } \\
(1975)\end{array}$ & 5.1 & -0.1 & 4.3 & 60.89 & 1.49 & 69.23 \\
\hline $\begin{array}{l}\text { Terasaki et al. } \\
\text { (1979) }\end{array}$ & 5.12 & -0.13 & 4.34 & 60.79 & 1.95 & 69.60 \\
\hline $\begin{array}{l}\text { Shishido } \\
\text { et al. (1975) }\end{array}$ & - & -0.12 & - & & & \\
\hline $\begin{array}{l}\text { present } \\
\text { result }\end{array}$ & 5.12 & -0.15 & 4.40 & 60.80 & 2.23 & 69.00 \\
\hline
\end{tabular}

mentioned above, the sign of the 222 structure potential has to be negative as determined in our present work and in a preliminary result. In the first publication [7] we made a mistake in converting this structure potential to the $\mathrm{x}$-ray value concerning the sign. Dr. Terasaki drew our attention to this point. The mean values of the converted scattering factors of Terasaki et al. [15] are in good agreement with the present results. The small deviation of our first results from the present analysis was due to the fact that absorption was neglected and only systematic interactions were considered. Furthermore the accuracy of the analysis was not as good, because the curve fitting procedure was done only with the single reflections, excluding the zero beam, and not with the continous densitometer record of all systematic reflections, as emphasized in a previous chapter.

During the analysis it was found that the accuracy for determining the structure potentials depends on the crystal thickness. For thicknesses, where the intensity of the reflection is very small due to "Pendellösung" effects, i. e. thicknesses ap- proximately equal to $(n+1 / 2)$ of the extinction length $(n=0,1,2, \ldots)$, the calculated intensity profile is most sensitive to the structure potential. In this case the background can be determined with high accuracy. In this thickness region the reflection intensity is also very sensitive to the thickness. The strong dependence of intensity on thickness has been used in the electron microscope to obtain images of atomic steps on single crystal surfaces [19]. In the convergent beam experiment the crystal thickness is determined from the intensity distribution in the weak Kossel-Möllenstedt reflections within an accuracy of a lattice constant, which means $4-5 \AA$. Thus the structure potentials can be determined at an appropriate thickness with very high accuracy.

The mean absorption was determined by measuring the current of the incident electron beam and the current density distribution in the diffracted beams taking into consideration the crystal thickness. The absorption potentials were calculated from relation (3) using the parameters $B$ and $C$. The results are shown in Table 5 together with other experimental data and the theoretical values due to Radi [20]. The present values, which are very reliable, are very small and about $1 / 3$ of the theoretical values.

\section{Summary}

By the convergent beam electron diffraction technique low-indexed structure potentials can be determined with high accuracy from very small crystal areas of the order of $100 \AA$ in diameter. Problems due to an inelastic background in the diffraction pattern as well as the specimen contamination could be overcome. An influence of doping on the 111-structure potential of silicon was found. The accurate determination of crystal thickness of the order of $4 \AA$ allowed the determination of the mean absorption potential. To achieve high accuracy in

Table 5. Absorption potentials of silicon (in volts.)

\begin{tabular}{lllll}
\hline$h k l$ & $\begin{array}{l}\text { pre- } \\
\text { sent } \\
\text { result }\end{array}$ & $\begin{array}{l}\text { Ichimiya and } \\
\text { Lehmpfuhl } \\
(1978)\end{array}$ & $\begin{array}{l}\text { Kreutle and } \\
\text { Meyer-Ehmsen } \\
(1969) \\
500^{\circ} \mathrm{K}\end{array}$ & $\begin{array}{l}\text { Radi } \\
(1970)\end{array}$ \\
\hline 000 & 0.61 & - & 0.92 & 0.70 \\
111 & 0.031 & 0.044 & 0.15 & 0.1 \\
220 & 0.039 & 0.052 & - & 0.11 \\
311 & 0.025 & 0.032 & - & 0.07 \\
004 & 0.027 & 0.038 & - & 0.09 \\
$B$ & 0.004 & 0.0055 & - & - \\
$C$ & 0.0003 & 0.00045 & - & - \\
\hline
\end{tabular}


structure potential determination an adequate thickness has to be chosen, which should be near $1 / 2$ of the extinction length.

\section{Acknowledgement}

We want to express our gratitude to Prof. Dr. K. Molière for his interest and valuable suggestions. We thank Dr. K. Kambe for many fruitful discus-

[1] P. Goodman and H. J. Whitfield, Acta Cryst. A36, 219 (1980).

[2] T. Shishido and M. Tanaka, Phys. stat. sol. (a) 38, 453 (1976).

[3] J. W. Steeds, G. J. Tatlock, and J. Hampson, Nature London. 241, 435 (1973).

[4] H. Bethe, Ann. Phys. 4. Folge, 87, 55 (1928).

[5] J. M. Cowley and A. F. Moodie, Proc. Phys. Soc. 71, 533 (1957).

[6] P. J. E. Aldred and M. Hart, Proc. Roy. Soc. London A332, 223 (1973).

[7] P. J. Smith and G. Lehmpfuhl, Acta Cryst. A31, S 220 (1975).

[8] G. Lehmpfuhl and A. Reissland, Z. Naturforsch. 23 a, 544 (1968).

[9] K. Moliére, Ann. Phys. 5. Folge, 34, 461 (1939).

[10] P. Doyle and A. Turner, Acta Cryst. A 24, 390 (1968).

[11] C. J. Humphreys and P. B. Hirsch, Phil. Mag. 18, 115 (1968). sions, and we thank Mr. E. Schumann for constructing and refining the convergent beam diffraction camera. We are also indebted to Dr. D. Krahl for his help in changing the dimensions of the objective pole-piece and to Dr. P. L. Fejes for providing us with the computer program for plotting the Kikuchi lines. Two of us (R.V. and P. J.S.) whish to thank the Max-Planck-Gesellschaft for financial support.

[12] A. Ichimiya and G. Lehmpfuhl, Z. Naturforsch. 83 a, 269 (1978)

[13] G. Lehmpfuhl, IX. International Congress on Electron Microscopy, Toronto, 1978 Vol III, 304.

[14] G. Lehmpfuhl and P. J. Smith, BEDO 8, 347 (1975), R. A. Remy-Verlag, Münster 1977.

[15] O. Terasaki, D. Watanabe, and J. Gjønnes, Acta Cryst. A 35, 895 (1979).

[16] M. Kreutle and G. Meyer-Ehmsen, Phys. stat. sol. 35, K17 (1969).

[17] Y. Ando, A. Ichimiya, and R. Uyeda, Acta Cryst. A 30, 600 (1974).

[18] T. Shishido and M. Tanaka, Japan J. Appl. Phys. 14, 135 (1975).

[19] G. Lehmpfuhl and Y. Uchida, Ultramicroscopy 4, 275 (1979).

[20] G. Radi, Acta Cryst. A 26, 41 (1970).

[21] P. Goodman and G. Lehmpfuhl, Acta Cryst. 22, 14 (1967). 